\title{
Contention Tree-based Access for Wireless Machine-to-Machine Networks with Energy Harvesting
}

\author{
F. Vázquez-Gallego*, C. Kalalas*, L. Alonso ${ }^{\dagger}$, and J. Alonso-Zarate* \\ ${ }^{*}$ Centre Tecnològic de Telecomunicacions de Catalunya (CTTC), Castelldefels \\ Barcelona, Spain. \\ \{francisco.vazquez, ckalalas, jesus.alonso\}@cttc.es \\ ${ }^{\dagger}$ Universitat Politècnica de Catalunya (UPC), Spain. \\ luisg@tsc.upc.edu
}

\begin{abstract}
In this paper, we consider a wireless Machine-toMachine network composed of end-devices with energy harvesters that periodically transmit data to a gateway. While energy harvesting allows for perpetual operation, the uncertain amount of harvested energy may not guarantee fully continuous operation due to temporary energy shortages. This fact needs to be addressed at the Medium Access Control (MAC) layer. We thus investigate the performance of an Energy Harvestingaware Contention Tree-based Access (EH-CTA) protocol, which uses a tree-splitting algorithm to resolve collisions and takes energy availability into account. We derive a theoretical model to compute the probability of delivery and the time efficiency. In addition, we conduct a performance comparison of EH-CTA using an EH-aware Dynamic Frame Slotted-ALOHA (EH-DFSA) as a benchmark. We determine the parameters that maximize performance and analyze how it is influenced by the amount of harvested energy and the number of end-devices. Results reveal the superior performance of EH-CTA over EH-DFSA. While EHDFSA requires an estimate of the number of contending enddevices per frame to adapt the frame length, EH-CTA uses short and fixed frame lengths, which enables scalability and facilitates synchronization as the network density increases.
\end{abstract}

Index Terms-Machine-to-Machine networks; contention; energy-harvesting capabilities; medium access control; treesplitting algorithm.

\section{INTRODUCTION}

Wireless Machine-to-Machine (M2M) networks aim at interconnecting and providing end-devices, e.g., sensors or actuators, with Internet connectivity. In many cases, these enddevices must operate autonomously for years with none or very limited access to energy sources. Two complementary strategies to extend the network lifetime consist in reducing the energy consumption required for communications and using energy harvesters [1] that collect energy from the environment. Despite the fact that energy harvesters can theoretically provide infinite lifetime, the high variability and unpredictability in the energy harvesting processes may not guarantee fullycontinuous operation. For example, the available energy over a short period of time may not be enough for the operation of an end-device, which may enter temporarily in energy shortage. This fact needs to be considered for the design of Medium Access Control (MAC) protocols which make an efficient use of the available energy and take the energy-harvesting process into account. Motivated by this need, in this paper we focus on data collection scenarios using wireless M2M area networks where hundreds or thousands of end-devices equipped with energy-harvesters periodically transmit data upon request from a gateway. While the traffic load generated by each enddevice may be low, the number of end-devices that attempt simultaneously to get access to the wireless channel can be potentially large, thus posing a challenge to the design of the MAC layer.

In dense M2M networks with a large and dynamic number of end-devices, Time Division Multiple Access (TDMA) is not optimal due to the delay and energy required to update the knowledge of the network topology and to maintain a collision-free schedule. On the contrary, the simplicity of random-access protocols, such as ALOHA or Carrier Sense Multiple Access (CSMA), makes them ideal for wireless networks with a dynamic number of simple end-devices. In fact, most standards typically used in M2M area networks, e.g., IEEE 802.11, IEEE 802.15.4, ISO/IEC-18000-7, rely on random-access protocols. Unfortunately, when the traffic load increases or the number of simultaneous contending end-devices is high, random access is prone to suffer from degraded performance due to uncontrolled congestion [2].

A possible strategy to counteract congestion and improve the performance of random access is to use a Collision Resolution Algorithm (CRA). The basic CRA relies on the tree-splitting algorithm [3], also referred to as Contention Tree Algorithm (CTA). This algorithm resolves the collisions by organizing the retransmission of colliding data packets in such a way that collisions are split and reduced, and all the packets can be successfully transmitted with finite delay and energy consumption. The performance of tree-splitting algorithms has been evaluated in different applications such as Radio Frequency Identification (RFID) [4]-[6] and sensor networks [7]. Results show that there is a short and fixed frame length that minimizes delay and energy consumption regardless of the number of end-devices.

Since their introduction, random access protocols along with several proposed variations have been investigated very extensively in the literature. However, the performance analyses of random access protocols on networks with energy 
harvesting is a much more recent area of research. Existing works focus on the assessment of slotted-ALOHA [8], CSMA [9] [10], Frame Slotted-ALOHA (FSA) [11], and Dynamic Frame Slotted-ALOHA (DFSA) [12]-[14]. However, to the best of the authors' knowledge, the design and analysis of random access protocols based on tree-splitting for wireless networks comprised of energy-harvesting nodes has never received attention.

Recently, the authors proposed in [15] a novel MAC protocol, coined Energy Harvesting-aware Contention Tree-based Access (EH-CTA), in an attempt to exploit the potentials of tree-splitting on wireless M2M networks with energy harvesting capabilities. EH-CTA uses an $m$-ary tree-splitting algorithm based on a Collision Resolution Queue (CRQ) to resolve collisions while accounting for the energy availability. Focusing on data-collection scenarios, the authors evaluate the performance of EH-CTA in terms of the frame length for different energy harvesting rates and numbers of end-devices. Motivated by the promising results, in this paper we extend the analysis of EH-CTA with the following contributions:

1) Using a Markov chain model to analyze the evolution of the available energy in an end-device, we determine the configuration parameters of EH-CTA that maximize $(i)$ the probability of delivery, which measures the ability of the MAC protocol to successfully transmit data to the gateway from the end-devices without depleting their energy reserves, and (ii) the time efficiency (or data collection rate), which measures the average number of data packets received by the coordinator per time slot. We further validate our proposed mathematical framework with the aid of computer-based simulations.

2) We reveal and study the fundamental trade-off between the probability of delivery and the time efficiency and we investigate how both performance metrics are influenced by the energy threshold, the energy harvesting rate and the number of end-devices.

3) We highlight the superior performance of EH-CTA over an EH-aware DFSA (EH-DFSA) protocol for a different number of end-devices present in the network. By capitalizing on the performance gains of EH-CTA, we provide key insights for the design of energy-efficient MAC protocols with $\mathrm{EH}$ CTA in large-scale data-collection scenarios.

The remainder of this paper is organized as follows. In Section II, we describe the system model. In Section III and Section IV, we summarize the operation of the EHCTA and EH-DFSA protocols and the performance metrics, respectively. In Section $\mathrm{V}$, we present the analytical model. Section VI is devoted to evaluate the performance of EH-CTA and validate the accuracy of the analysis through comprehensive computer-based simulations. The performance of EH-CTA and EH-DFSA are also compared in Section VI. Finally, our concluding remarks are presented in Section VII.

\section{SySTEM MODEL}

\section{A. Network and Data Model}

We consider a single-hop wireless M2M area network where communication is established between $n$ end-devices and a central coordinator (also referred to as gateway). Each enddevice is equipped with an energy harvester and an energystorage device (ESD), i.e., rechargeable battery or supercapacitor, to store the energy captured by the energy harvester. As illustrated in Figure 1, the coordinator is responsible for the data collection process from the end-devices which occurs once every $T_{R}$ seconds, in periodic Data Collection Rounds (DCR). In particular, a DCR is initiated when the coordinator broadcasts a Request for Data (RFD) packet. The RFD of the $k$-th DCR is followed by a sequence of $F_{k}$ frames further divided into $m$ equal time slots. We assume that in each DCR, an end-device has exactly a new data packet ready to transmit to the coordinator. The size of the data packet is fixed and its duration fits within the duration of a slot.

An end-device enters into active mode when the available energy in its ESD is higher than a certain threshold at the beginning of a DCR. On the contrary, if the available energy does not exceed the threshold, the end-device remains in sleep mode waiting for the next DCR. In active mode, communication is performed and the end-device attempts to transmit its data packet following the MAC protocol discipline, as detailed in Section III. We assume that the active end-devices select a random slot to transmit without performing carrier sensing or Clear Channel Assessment (CCA). As shown in Figure 1, the coordinator transmits a feedback packet (FBP) at the end of each frame that includes information about the time slots' status. From the perspective of the coordinator, every slot in a frame can be in one of three states: (i) success, i.e., a data packet sent from an end-device is correctly received and decoded; (ii) failure, i.e., one or more transmitting end-devices have selected the slot but no data packet can be decoded by the coordinator due to a collision or channel error; or (iii) empty, i.e., no data packet is received and the channel is idle.

When an end-device learns from the FBP that its transmission was successful in a given frame, it enters into sleep mode for energy saving until the next DCR initiation. Otherwise, if transmission fails in a given frame, the end-device will attempt to re-transmit its data packet in subsequent frames as long as its energy reserves are not depleted. In the example of Figure 1, end-devices 1, 2, and 4 have enough energy to contend in the $k$-th DCR, while end-device 3 is in energy shortage at the beginning of the DCR. In the subsequent $(k+1)$-th DCR, end-device 1 is in energy shortage, while end-devices 2, 3,

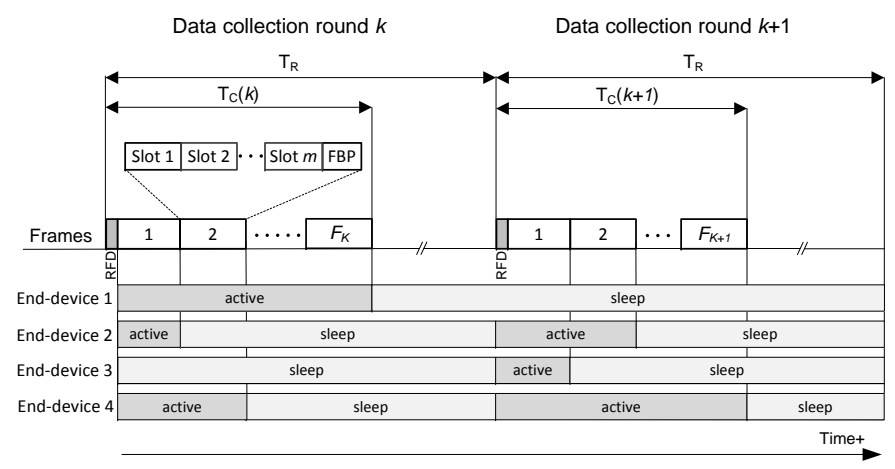

Figure 1: Illustration of the data collection process [15]. 
and 4 have enough energy to contend. We consider that if an end-device fails to transmit its data packet due to energy shortage in any given DCR, the data packet is discarded and it is not transmitted in subsequent DCRs. We leave for future work the performance evaluation considering that the packets not transmitted in a DCR are buffered to be sent in the next DCRs.

The contention process is terminated when all the enddevices have entered into sleep mode due to either successful transmission or energy shortage. We assume that the contention time, $T_{c}(k)$, elapsed since the $k$-th DCR initiates until the contention process terminates, is much shorter than the time between two consecutive DCRs, i.e., $T_{c}(k) \ll T_{R}$ for all $k$, in order to ensure that successive DCRs do not overlap. Since in this work we focus our attention on the MAC layer, we assume that all packets are always transmitted without errors induced by the wireless channel. In addition, we assume that none of the data packets involved in a collision can be decoded by the coordinator, i.e., we neglect the capture effect. The inclusion of transmission errors and capture effect constitutes part of our future work.

\section{B. Energy Storage and Energy Consumption Models}

In this work, we consider that the amount of energy stored in the ESD of an end-device is modeled as a random variable which depends on the harvested energy and the energy consumed by the end-device during the DCRs. In particular, the available energy in the ESD of the $i$-th end-device is denoted by $E_{E S D, i} \in\{0,1 \delta, 2 \delta, \ldots, N \delta\}$, where $\delta$ [Joule] is referred to as energy unit and $N$ reflects the normalized capacity of the ESD. Let also $E_{t h r}=\varepsilon_{t h r} \delta$, with $\varepsilon_{t h r} \in\{0,1,2, \ldots, N-1\}$, denote the energy threshold. Then, the activation probability, $p_{\text {active }}(k)$, of an end-device entering in active mode and thus contending in the $k$-th DCR can be expressed as

$$
p_{\text {active }}(k)=\operatorname{Pr}\left\{E_{E S D, i}(k)>E_{t h r}\right\},
$$

where $E_{E S D, i}(k)$ denotes the stored energy of the $i$-th enddevice at the beginning of the $k$-th DCR. The energy threshold $\varepsilon_{t h}$ must be selected so as to maximize the probability that an end-device succeeds in transmitting its data packet to the coordinator in a DCR.

Energy consumption for an end-device occurs during all communication phases with the coordinator, i.e., (i) packet transmission in a slot, (ii) during sleep mode in the remaining $m-1$ slots of the frame, and (iii) while idle listening for an incoming FBP. In this work, the energy consumed by an end-device in sleep mode is considered negligible. We further assume that each time an end-device transmits its data packet in a certain frame of a DCR, it consumes a constant amount of energy, denoted by $E_{t x}$ [Joules], which accounts for the entire energy consumption during transmission and idle listening. For mathematical tractability, we normalize the energy consumption $E_{t x}$ to one energy unit, i.e., $E_{t x}=1 \delta$. Therefore, when the ESD of an end-device is fully charged at the beginning of a DCR, i.e., it contains $N$ energy units, the maximum number of data packet transmissions is $N$.

\section{Energy Harvesting Model}

For the time interval $T_{R}$ between any two consecutive, $(k-1)$-th and $k$-th, DCRs, we assume that the amount of energy, $E_{H, i}(k)$, captured by the energy harvester of the $i$ th end-device can be modeled as a discrete random variable with a probability mass function $q_{j}=\operatorname{Pr}\left\{E_{H, i}(k)=j \delta\right\}$ with $j \in\{0,1,2, \ldots\}$ energy units, which depends on the energy source characteristics. The stochastic process $E_{H, i}(k)$ is considered to be identically and independently distributed over all end-devices and DCRs. In addition, we define the energy harvesting rate, $\bar{E}_{H}$, as the average energy harvested by an end-device during the time $T_{R}$ between the beginning of two consecutive DCRs, i.e.,

$$
\bar{E}_{H}=\mathbb{E}\left[E_{H, i}(k)\right] .
$$

The dynamics of the energy harvesting process is considered slower than the contention process within a DCR. Based on this assumption, the amount of harvested energy during $T_{c}(k)$ can be considered negligible with respect to $E_{H, i}(k)$, and it is not immediately available for use during the contention process. Thus, all the harvested energy $E_{H, i}(k)$ is available to be used by an end-device at the beginning of the $k$-th DCR.

\section{MAC PROTOCOLS}

\section{A. Energy Harvesting-aware Dynamic Frame Slotted-ALOHA}

The DFSA protocol for data collection networks with energy harvesting nodes (also referred to as EH-DFSA) was first presented in [14] and further analyzed in [16]. In EH-DFSA, the contention process in each DCR is composed of a sequence of frames further divided into a variable number of slots. The number of slots is dynamically adjusted to be equal to the estimated number of end-devices that contend in each frame in order to minimize $T_{C}(k)$ [14], [17]. An end-device that becomes active in a DCR randomly selects one of the slots in every frame to transmit its data packet. When an end-device either succeeds in transmitting its data packet or falls in energy shortage in a given frame, it enters into sleep mode and stops contending again in subsequent frames of the same DCR. In any other case, it re-attempts data packet transmission only if its available amount of energy allows for it. In turn, the coordinator estimates the number of contending end-devices in the subsequent frame and adaptively broadcasts the number of available slots in the FBP. An estimation algorithm is proposed in [14].

\section{B. Energy Harvesting-aware Contention Tree-based Access Protocol}

The EH-CTA constitutes the MAC protocol proposed and investigated in this work. It implements a tree-splitting algorithm and takes into account the energy available in the ESDs. In EH-CTA, the end-devices which become active in a DCR are organized into sub-groups using a tree-splitting algorithm, which reduces the probability of collision per transmission attempt. As illustrated in Figure 2.a., the algorithm can be represented by a contention tree, where each node of the tree corresponds to a time frame with a fixed number $m$ of slots. 


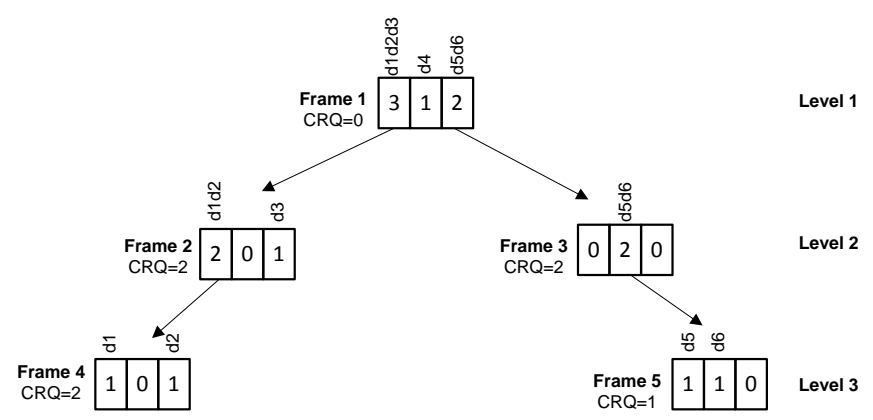

(a) Tree-splitting algorithm

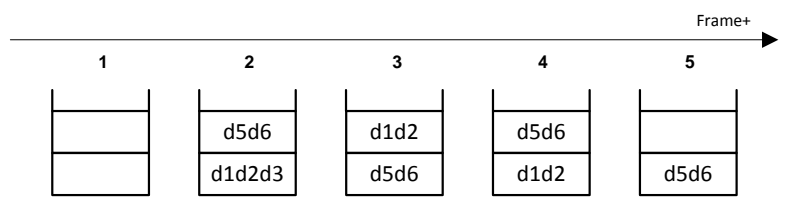

(b) Contents of CRQ in each frame of the process

Figure 2: Example of data collection round using EH-CTA with $n=6$ end-devices (d1 to d6) that become active at the beginning of the DCR, $m=3$ slots per frame, and $N \geq 3$.

The number included in every slot of a frame denotes the number of end-devices that transmit in the specific slot.

The tree-splitting algorithm resolves the contention in subgroups as follows. In the first frame, all end-devices that are in active mode randomly select a slot to send their data packet. In case that two or more end-devices select the same slot, a collision occurs. An additional frame for retransmission is then solely allocated to the sub-group of the collided enddevices which are queued into a logical queue referred to as the Collision Resolution Queue (CRQ). Accordingly, if there are $k$ slots with collision in a frame of level $d$ of the tree, with $d \in\{1,2, \ldots, N\}$, then $k$ new frames are scheduled in level $d+1$, and $k$ sub-groups of end-devices are queued into the CRQ. Figure 2.a. shows the tree-splitting algorithm execution, considering an example of 6 active end-devices and 3 contention slots in a frame. This iterative process leads to the formation of a tree whose expansion stops at frames with only successful and/or empty slots, meaning that all collisions have been resolved, or at level $N$, which is the normalized capacity of the ESD and thus limits the number of levels where an enddevice can re-transmit its data packet. An end-device transmits its data-packet in a frame of every level $d$ of the tree until it succeeds in one level or enters in energy shortage.

The CRQ is represented at each end-device by two integer numbers: $(i)$ the position of the end-device in the CRQ, and (ii) the total length of the CRQ, which represents the number of sub-groups of end-devices waiting to re-transmit. Using the information (contention slot status, CRQ length) included in the broadcast FBP at the end of each frame, an end-device that has collided in a given frame can compute its position in the CRQ. The position of an end-device in the CRQ is always decreased by one unit at the end of each frame to account for the frame execution. If the end-device occupies the first position in the CRQ, packet retransmission occurs in the next frame selecting a random slot; otherwise, it waits for its turn without contending during successive frames. Therefore, an end-device only needs to receive the FBP of those frames where it contends in order to know whether it has succeeded (and then leaves the CRQ) or has collided (and then enters into the CRQ again).

An example of the evolution of the contents of the CRQ is shown in Figure 2.b. At frame 1, all the end-devices that become active (referred to as $\mathrm{d} 1, \mathrm{~d} 2, \ldots \mathrm{d} 6$ ) transmit their data packet: $\mathrm{d} 1, \mathrm{~d} 2$ and $\mathrm{d} 3$ collide in slot 1 and enter in the first position in the CRQ; d5 and d6 collide in slot 3 and enter in the second position; and $\mathrm{d} 4$ succeeds in slot 2 . At frame 2, $\mathrm{d} 1, \mathrm{~d} 2$ and $\mathrm{d} 3$ contend because they occupy the first position in the CRQ: $\mathrm{d} 1$ and $\mathrm{d} 2$ collide and enter again in the CRQ; $\mathrm{d} 3$ succeeds and leaves the CRQ; and d5 and d6 move to the first position in the CRQ, thus, $\mathrm{d} 5$ and $\mathrm{d} 6$ do not contend in frame 2. At frame 3, d5 and d6 contend because they occupy the first position in the CRQ, they collide and enter again in the CRQ; and $\mathrm{d} 1$ and $\mathrm{d} 2$ do not contend and move to the first position in the CRQ. At frame 4, d1 and d2 contend and succeed; and $\mathrm{d} 5$ and $\mathrm{d} 6 \mathrm{do}$ not contend and move to the first position in the CRQ. Finally, d5 and d6 contend and succeed at frame 5.

\section{Performance Metrics}

Before the analysis, we first introduce the two performance metrics that have been used to assess the considered MAC protocols, namely:

1) The probability of delivery, defined as the probability that an end-device enters into active mode in the $k$-th DCR and succeeds in transmitting its data packet to the coordinator. An end-device fails to transmit data in a DCR if it enters in energy shortage before its data packet is successfully transmitted. Thus, the probability of delivery reflects the ability of the MAC protocol to successfully deliver data from an end-device to the coordinator in a DCR without depleting its ESD.

2) The time efficiency, defined as the ratio between the number of data packets successfully transmitted to the coordinator in the $k$-th DCR, and the total number of slots required to complete the DCR. This value measures the average number of data packets received per time slot in a DCR. Therefore, it constitutes an indicator of the data collection rate, which can be obtained by dividing the time efficiency by the duration of a slot.

Since the use of energy harvesters potentially allows for perpetual operation, it is interesting to analyze the performance metrics when the system is in steady-state, i.e., for a DCR with large index $k$.

\section{Analysis of Performance Metrics}

In order to derive an analytical model to calculate the performance metrics in steady-state for EH-CTA, we first need to compute the steady-state probability distribution of the available energy in the ESDs at the beginning of a DCR, which depends on the energy harvesting process, the random slot selection in every frame and the tree splitting process. Given that the number of contending end-devices in every 
level of the contention tree depends on the energy available in the ESD at each end-device, deriving the exact steady-state probability distribution is not a trivial task. As an example, an exact stochastic model to analyze the energy evolution at the ESDs would require a set of interconnected Markov chains (one for each end-device).

However, if we adjust the value of the energy threshold $\varepsilon_{t h}$ (1) to guarantee that all the end-devices that enter in active mode $\left(n_{1}\right)$ in a DCR will have enough energy to contend in a certain number of levels, assuming that the number of enddevices falling in energy shortage in a DCR is negligible, we can consider that the probability that an end-device succeeds in transmitting its data packet in one frame of any level of the contention tree basically depends on the value of $n_{1}$, the number of slots per frame, and the number of level. Consequently, we can compute the steady-state probability distribution of the available energy in the ESDs by simply analyzing the evolution of the energy in a single ESD, which is an approximation that neglects the interactions among the ESDs of different end-devices.

\section{A. Markov Chain Model}

Based on the previous considerations, a discrete-time Markov chain can be used to model the evolution of the available energy in the ESD of an end-device, as illustrated in Figure 3. Let $e(t) \in\{0,1, \ldots, N\}$ be a stochastic process which represents the number of energy units stored in the ESD at time $t$. Let also $d(t) \in\{0,1, \ldots, N\}$ be a stochastic process which represents that either an end-device is in sleep mode when $d(t)=0$, or the level in the contention tree in which an end-device contends when $d(t) \in\{1, \ldots, N\}$. Each state of the Markov chain is then defined as $\{e(t), d(t)\}$. Note that in every level $d$ of the tree, an end-device transmits its data packet in only one frame while the state transitions in the discrete-time Markov chain do not occur at fixed time intervals.

Let $\mathrm{P}=\left[p_{i j}\right]$ be the state transition matrix of the finite state space of the Markov chain. Each element $p_{i j}$ corresponds to the one-step transition probability defined as

$$
\operatorname{Pr}\left\{e(t+1)=e_{j}, d(t+1)=d_{j} \mid e(t)=e_{i}, d(t)=d_{i}\right\} .
$$

As discussed in Section II-A, in case the end-device succeeds or enters in energy shortage in a DCR, it remains in sleep mode (i.e., in one of the states with $d_{i}=0$ ) until the next DCR. Each time a DCR initiates, the harvested energy $\varepsilon_{H}$ during the previous $T_{R}$ interval is accumulated to the ESD energy deposits, i.e., $e_{j}=e_{i}+\varepsilon_{H}$. We denote by $q_{\varepsilon_{H}}$ the probability that an end-device harvests $\varepsilon_{H} \in\{0,1,2, \ldots\}$ energy units. According to the energy storage model introduced in Section II-B, the end-device remains in sleep mode if the available energy in its ESD is less or equal to the threshold $\varepsilon_{t h}$, i.e., $e_{j} \in\left\{0,1, \ldots, \varepsilon_{t h}\right\}$. This is represented in the Markov chain with a state transition from $\left(e_{i}, 0\right)$ to $\left(e_{j}, 0\right)$. On the other hand, the end-device enters in active mode if $e_{j} \in\left\{\varepsilon_{t h}+1, \ldots, N\right\}$, and a state transition occurs from $\left(e_{i}, 0\right)$ to $\left(e_{j}, 1\right)$.

The state transition probability from state $\left(e_{i}, 0\right)$ to any state

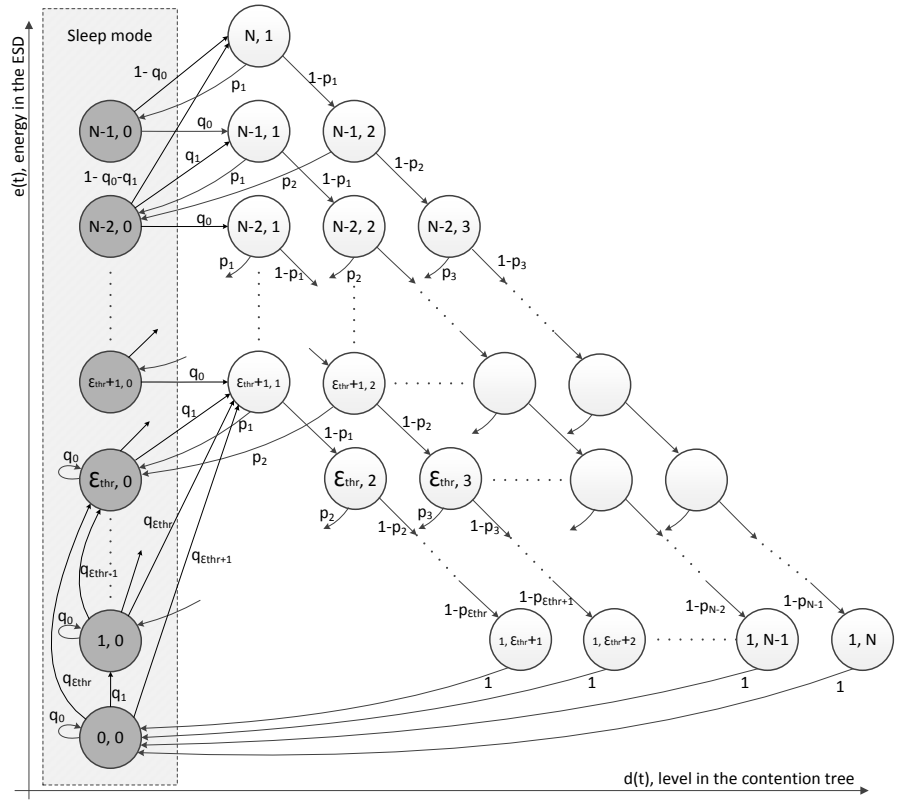

Figure 3: Generalized state-transition diagram of the discretetime Markov chain used to model the evolution of the available energy in the ESD of an end-device. Some arcs representing transitions between states have been intentionally omitted to facilitate understanding.

$\left(e_{j}, d_{j}\right)$ at the beginning of a DCR can then be expressed as

$$
p_{i j}=\left\{\begin{array}{lr}
q_{\varepsilon_{H}}, & i f\left(e_{j} \leq \varepsilon_{t h}\right) \text { and }\left(d_{j}=0\right) \\
q_{\varepsilon_{H}}, & \text { if }\left(\varepsilon_{t h}<e_{j}<N\right) \text { and }\left(d_{j}=1\right) \\
1-\sum_{k=0}^{N-1-e_{i}} q_{k}, & \text { if }\left(e_{j}=N\right) \text { and }\left(d_{j}=1\right) \\
0, & \text { otherwise }
\end{array}\right.
$$

Following the rules of EH-CTA as described in Section III-B, if the end-device enters in active mode, it re-transmits its data packet in a frame of every successive level of the contention tree until either it succeeds or its ESD falls in energy shortage. The successful transmission in a frame of level $d_{i} \in\{1, \ldots, N\}$ is represented by a transition from state $\left(e_{i}, d_{i}\right)$ to state $\left(e_{i}-1,0\right)$ for $e_{i} \in\{1,2, \ldots, N\}$. Otherwise, i.e., in the case of unsuccessful transmission, the end-device can make two possible state transitions: $(i)$ to state $\left(e_{i}-1, d_{i}+1\right)$ if its available energy deposits are adequate for retransmission in a frame of the next level of the tree, i.e., $e_{j} \in\{1,2, \ldots, N-1\}$; or (ii) to state $(0,0)$ if energy shortage occurs, i.e., $e_{j}=0$.

Let $p_{d}$ be the probability of successful transmission from an end-device in one frame of level $d=d_{i}$. Then, the state transition probability from $\left(e_{i}, d_{i}\right)$ to $\left(e_{j}, d_{j}\right)$ with $d_{i} \in\{1,2, \ldots, N\}$ can be expressed as

$$
p_{i j}=\left\{\begin{array}{lr}
p_{d}, & i f\left(e_{j}=e_{i}-1\right) \text { and }\left(d_{j}=0\right), \\
1-p_{d}, & \text { if }\left(e_{j}=e_{i}-1\right) \text { and }\left(d_{j}=d_{i}+1\right), \\
1, & \text { if }\left(e_{i}=1\right) \text { and }\left(e_{j}=0\right) \text { and }\left(d_{j}=0\right), \\
0, & \text { otherwise }
\end{array}\right.
$$

In the following subsection, we derive the expression of $p_{d}$. 


\section{B. Probability of Success in one Frame}

Let $m$ denote the number of slots per frame and $n_{d}$ be the number of contending end-devices in one frame of level $d$. Then, considering the viewpoint of an end-device, the probability of successful transmission in a frame of level $d$ can be expressed as

$$
p_{d}=\left(1-\frac{1}{m}\right)^{n_{d}-1} \text { for } d \geq 1
$$

In the first frame of a steady-state DCR (index $k \rightarrow \infty$ ), the number of contending end-devices in level $d=1, n_{1}$, is determined by the average number of end-devices in active mode, i.e., $n_{1}=n \cdot p_{\text {active }}^{S S}$, where $n$ denotes the total number of end-devices in the network and $p_{\text {active }}^{S S}$ is the activation probability in steady-state. Based on the definition of $p_{\text {active }}(k)$ in (1), $p_{\text {active }}^{S S}$ is given by

$$
p_{\text {active }}^{S S}=\lim _{k \rightarrow \infty} p_{\text {active }}(k) .
$$

In order to ensure that an end-device entering in active mode in a DCR has enough energy to contend until the successful data packet transmission, the energy threshold $\varepsilon_{t h}$ must be configured accordingly. In this paper, we assume that $\varepsilon_{t h}$ is set to an appropriate value that guarantees that the number of active end-devices that deplete their energy deposits during a DCR is negligible. Under this assumption, the value of $n_{d}$ for $d>1$ can be calculated as follows. First, the probability $p_{s}(k)$ that $k$ over $n_{d}$ end-devices transmit in the same slot of a frame can be expressed as

$$
p_{s}(k)=\left(\begin{array}{c}
n_{d} \\
k
\end{array}\right)\left(\frac{1}{m}\right)^{k}\left(1-\frac{1}{m}\right)^{n_{d}-k},
$$

while the average number of empty, success, and collision slots in the specific frame, denoted by $S_{d}^{E}, S_{d}^{S}$, and $S_{d}^{C}$, respectively, can be calculated as

$$
\begin{gathered}
S_{d}^{E}=m \cdot p_{s}(0)=m\left(1-\frac{1}{m}\right)^{n_{d}}, \\
S_{d}^{S}=m \cdot p_{s}(1)=n_{d}\left(1-\frac{1}{m}\right)^{n_{d}-1}, \\
S_{d}^{C}=m-S_{d}^{E}-S_{d}^{S} .
\end{gathered}
$$

According to the tree-splitting algorithm of EH-CTA described in Section III-B, if there are $S_{d}^{C}$ (11) collision slots in one frame of level $d$, then $F_{d+1}=S_{d}^{C}$ additional frames are scheduled in the subsequent level $d+1$. Each additional frame is now assigned solely to the sub-group of end-devices that collided in the same slot of the previous level $d$. The average number of end-devices with successful transmission in one frame of level $d$, denoted by $n_{d}^{S}$, equals to the average number $S_{d}^{S}$ of slots with success, as expressed in (10). Hence, the average number of end-devices that collide in one frame of level $d$, denoted by $n_{d}^{C}$, can be calculated as $n_{d}^{C}=n_{d}-S_{d}^{S}$. Then, since the number of end-devices that fall in energy shortage in a DCR is assumed to be negligible, the $n_{d}^{C}$ enddevices will have enough energy to contend again in $F_{d+1}$ new frames of the subsequent level $d+1$. Therefore, the average

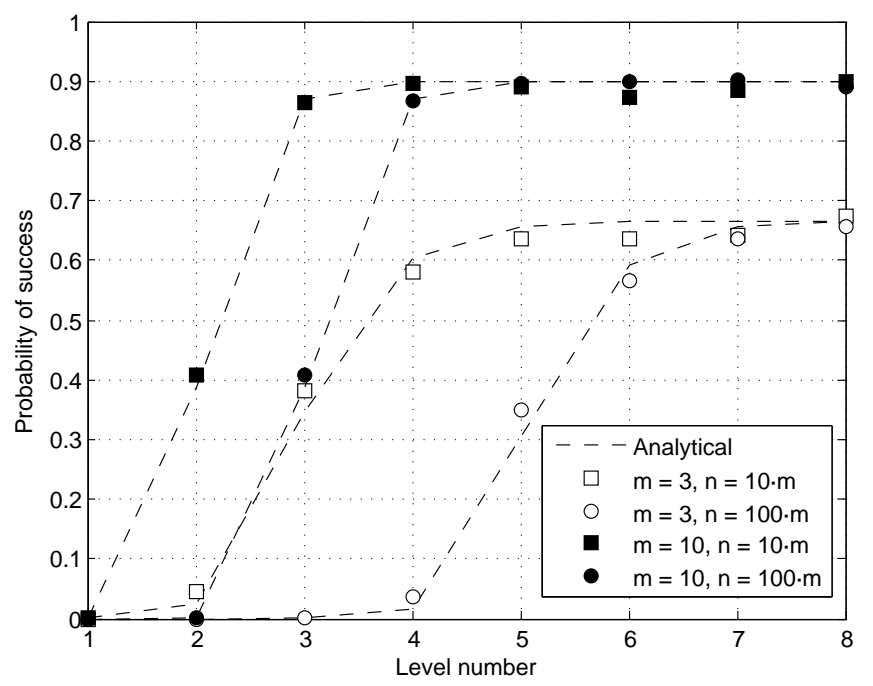

Figure 4: Probability of success in one frame of every level of the contention tree.

number of end-devices that contend in one frame of level $d+1$, denoted by $n_{d+1}$, can be approximated by the average number of end-devices $n_{d}^{C}$ that collide in one frame of level $d$ divided by the average number of frames $F_{d+1}$ scheduled in level $d+1$ from the frame in level $d$ where the $n_{d}^{C}$ end-devices collided. Finally, the average number of contending end-devices in one frame of level $d+1$ can be expressed as

$$
\begin{gathered}
n_{d+1} \simeq \frac{n_{d}^{C}}{F_{d+1}}=\frac{n_{d}^{C}}{S_{d}^{C}}, \\
n_{d+1} \simeq \frac{n_{d}-n_{d}\left(1-\frac{1}{m}\right)^{n_{d}-1}}{m-m\left(1-\frac{1}{m}\right)^{n_{d}}-n_{d}\left(1-\frac{1}{m}\right)^{n_{d}-1}} .
\end{gathered}
$$

Figure 4 illustrates the probability $p_{d}$ of successful transmission in one frame of every level of the contention tree, calculated using (6). It has been evaluated with $m \in\{3,10\}$, $n \in\{10 \cdot m, 100 \cdot m\}, p_{\text {active }}^{S S}=1$, and considering that all the end-devices that become active in a DCR have enough energy to contend until they succeed in transmitting their data packet in the DCR regardless of the energy harvesting rate and the capacity of the ESDs. Results show a tight match between analytical and simulated results. As it could be expected, the value of $p_{d}$ is close to 0 for low values of $d$, especially when the number $m$ of slots is low and the number $n$ of end-devices is high.

In order to determine the suitable value of the energy threshold $\varepsilon_{t h}$ in EH-CTA, i.e., the one that guarantees that an enddevice does not enter in energy shortage before it succeeds, we need to calculate the average number of frames, $\mathbb{E}[d]$, where an end-device has to contend for transmission until it finally succeeds in a DCR. With the aid of $p_{d}$ expression, the $\mathbb{E}[d]$ can be calculated as

$$
\mathbb{E}[d]=\sum_{d=1}^{\infty} d \cdot p_{d} \cdot \prod_{i=1}^{d-1}\left(1-p_{i}\right) .
$$




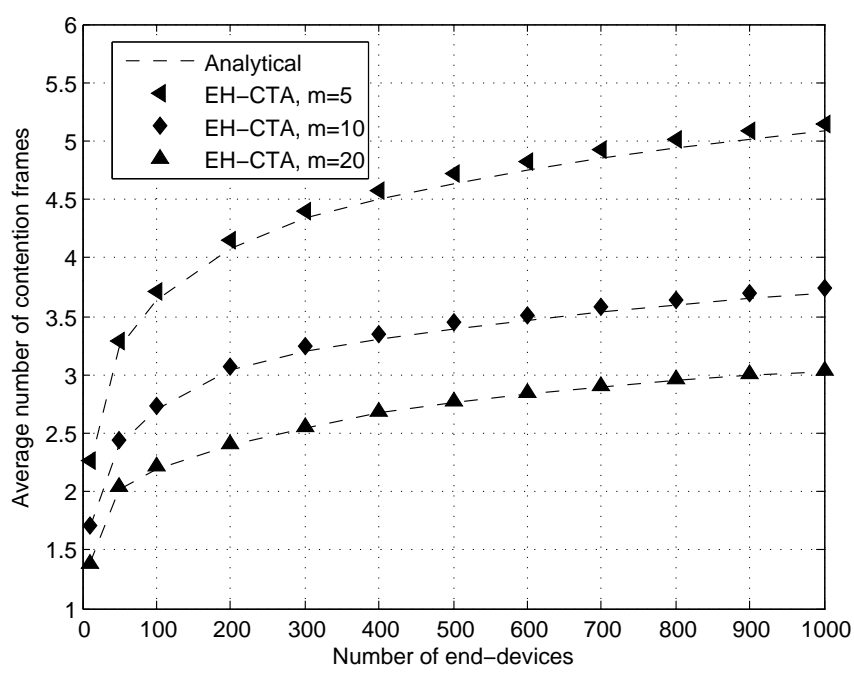

Figure 5: Average number of levels where an end-device contends until it succeeds in a DCR as a function of the number of end-devices.

Figure 5 depicts the value of $\mathbb{E}[d]$ as a function of the number $n$ of end-devices. It has been evaluated by considering $m \in\{5,10,20\}$. As it could be expected, the value of $\mathbb{E}[d]$ increases with increasing $n$ for a given value of $m$. The appropriate value of the energy threshold can thus be determined by setting it equal to the value of $\mathbb{E}[d]$, i.e., $\varepsilon_{t h} \approx \mathbb{E}[d]$, depending on the number of end-devices and slots. It can be observed from Figure 5 that in a network comprised of 1000 in total end-devices, the energy threshold $\varepsilon_{t h}$ must be close to 5 , 4, or 3 energy units when the number $m$ of slots is 5,10 , or 20 , respectively.

\section{Steady-State Probability Distributions}

According to the state transition regularities in Figure 3, when $p_{d}>0$ for $d \in\{1, \ldots, N\}, q_{0}>0$ and $q_{1}>0$, the discrete-time Markov chain is aperiodic and any state can be reached from any other state with non-zero probability. Hence, the Markov chain is irreducible.

Since the Markov chain is irreducible and aperiodic, and thus ergodic, it admits a unique steady-state probability distribution, $\pi=\left[\pi_{e, d}\right]$, which can be expressed as

$$
\pi_{e, d}=\lim _{t \rightarrow \infty} \operatorname{Pr}\{e(t)=e, d(t)=d\},
$$

and satisfies the set of linear equations,

$$
\left(\mathrm{P}^{\prime}-\mathrm{I}\right) \pi^{\prime}=0
$$

where $\mathrm{P}$ and I denote the state transition and identity matrices, respectively. Equation (16) can be solved for $\pi$ by computing the eigenvector of $\mathrm{P}^{\prime}$ that corresponds to an eigenvalue equal to 1 . The steady-state probability distribution $\pi$ is equal to the eigenvector with its elements normalized to sum one.

Recall that the transition matrix $\mathrm{P}$ depends on $p_{d}$ in (6), which in turn depends on $p_{\text {active }}^{S S}$ given by (7). On the other hand, $p_{\text {active }}^{S S}$ can be expressed from the steady-state probability distribution $\pi^{B}=\left[\pi_{e, d}^{B}\right]$ of the available energy in the ESD at the beginning of a DCR, as follows

$$
p_{\text {active }}^{S S}=\pi_{\varepsilon_{t h r}+1,1}^{B}+\ldots+\pi_{N, 1}^{B}=\sum_{e=\varepsilon_{t h r}+1}^{N} \pi_{e, 1}^{B} .
$$

Since an end-device is considered to be in sleep mode before a DCR initiates, $\pi^{B}$ can be expressed as

$$
\pi^{B}=\pi^{S} \mathrm{P}
$$

where $\pi^{S}=\left[\pi_{e, d}^{S}\right]$ represents the steady-state probability distribution conditioned on the end-device being in sleep mode, which is calculated as

$$
\pi_{e, d}^{S}=\left\{\begin{array}{lr}
\frac{\pi_{e, 0}}{\sum_{i=0}^{N-1} \pi_{i, 0}}, & \text { if }(d=0), \\
0, & \text { if }(1 \leq d \leq N) .
\end{array}\right.
$$

Finally, we compute the steady-state probability distributions using an iterative numerical method as follows. We set the steady-state activation probability to an initial test value of 0 , i.e., $p_{\text {active-test }}^{S S}=0$, and we calculate the transition matrix P. Next, we solve equations (16), (19), and (18) to calculate $\pi, \pi^{S}$, and $\pi^{B}$, respectively. Using $\pi^{B}$, we derive the analytical value of $p_{\text {active }}^{S S}$ in (17) and we check the relative error between the test and analytical value of the activation probability. This process is iteratively repeated with increasing $p_{\text {active-test }}^{S S}$ each time, until the relative error stands below $0.1 \%$, which indicates that it satisfies (16), (19) and (18), and the results obtained for $\pi, \pi^{S}$, and $\pi^{B}$ are correct.

We are now in position to derive the analytical expressions of the considered performance metrics, i.e., probability of delivery and time efficiency, in steady-state, as defined in Section IV. We provide the details in the following subsections.

\section{Probability of Delivery of EH-CTA}

Following the analysis of the previous subsection, once $\pi^{B}$ is computed, the steady-state probability of delivery for $\mathrm{EH}$ CTA, denoted by $p_{\text {delivery }}^{S S}$, can be calculated.

Note that if an end-device turns in active mode in a steadystate DCR, it starts in one of the states $(e, 1)$ with probability $\pi_{e, 1}^{B}$ for $e \in\left\{\varepsilon_{t h}+1, \ldots, N\right\}$. Then, it retransmits its packet in a frame of every level $d$ until it is correctly transmitted with probability $p_{d}$. Packet failure occurs when the end-device depletes its energy deposits before the packet is successfully decoded by the coordinator. Consequently, the expression of $p_{\text {delivery }}^{S S}$ is given by

$$
\begin{array}{r}
p_{\text {delivery }}^{S S}=\sum_{d=1}^{\varepsilon_{t h r}} \sum_{e=\varepsilon_{t h r}+1}^{N} \pi_{e, 1}^{B} \prod_{k=1}^{d-1}\left(1-p_{k}\right) p_{d}+ \\
\sum_{d=\varepsilon_{t h r}+1}^{N} \sum_{e=d}^{N} \pi_{e, 1}^{B} \prod_{k=1}^{d-1}\left(1-p_{k}\right) p_{d},
\end{array}
$$

where the first term of the summation corresponds to the probability that an end-device succeeds in levels $d \in\left\{1, \ldots, \varepsilon_{t h r}\right\}$ and the second term represents the probability that an enddevice succeeds in levels $d \in\left\{\varepsilon_{t h r}+1, \ldots, N\right\}$. As it can be observed in Figure 3, an end-device can succeed in levels 
$d \in\left\{1, \ldots, \varepsilon_{t h r}\right\}$ when it becomes active at the beginning of a DCR with an energy level $e \in\left\{\varepsilon_{t h r}+1, \ldots, N\right\}$, and it can succeed in levels $d \in\left\{\varepsilon_{t h r}+1, \ldots, N\right\}$ when its energy level is $e \in\{d, \ldots, N\}$ at the beginning of the DCR.

\section{E. Time Efficiency of EH-CTA}

The time efficiency of EH-CTA, $\eta_{t}$, can be expressed as the average number of slots with successful packet transmissions in a DCR divided by the total number of slots in this DCR. It is thus given by

$$
\eta_{t}=\frac{\sum_{d=1}^{N} \mathbb{E}\left[m_{S, d}\right]}{\sum_{d=1}^{N} \mathbb{E}\left[m_{d}\right]},
$$

where $\mathbb{E}\left[m_{d}\right]$ and $\mathbb{E}\left[m_{S, d}\right]$ denote the average number of contention and successful slots, respectively, in level $d \in$ $\{1, \ldots, N\}$. According to the expansion rule of the contention tree in EH-CTA, the number of collision slots, $S_{d}^{C}$ in (11), in a frame of level $d$ is equal to the number of additional frames to be scheduled in level $d+1$. By using the expression of the average number of frames in level $d$, which can be calculated as $\prod_{i=2}^{d} S_{i-1}^{C}$, and plugging it in (21), we get the following expression for the time efficiency of EH-CTA

$$
\eta_{t}=\frac{S_{1}^{S}+\sum_{d=2}^{N} S_{d}^{S} \prod_{i=2}^{d} S_{i-1}^{C}}{m+\sum_{d=2}^{N} m \prod_{i=2}^{d} S_{i-1}^{C}},
$$

where $m$ is the number of slots per frame, and $S_{d}^{S}$ and $S_{d}^{C}$ denote the average number of success and collision slots, respectively, in a frame of level $d$ which can be computed using (10) and (11).

In the next Section, we validate our proposed analytical model and evaluate the performance of EH-CTA in terms of probability of delivery and time efficiency. In addition, an insightful performance comparison of the EH-CTA and EH-DFSA protocols under various network configurations is presented.

\section{Vi. Model Validation and Performance EVALUATION}

To evaluate the performance of EH-CTA, we first describe the considered scenario and discuss the numerical results that reveal how the performance of the EH-CTA and EH-DFSA protocols is influenced by the following parameters: the energy threshold $\varepsilon_{t h r}$; the number $m$ of slots per frame in EH-CTA; the energy harvesting rate $\bar{E}_{H}$; and the total number $n$ of end-devices.

\section{A. Scenario}

We consider a wireless network topology that consists of 1 coordinator surrounded by a number $n$ of end-devices that reside within the transmission range of the coordinator. Each end-device is equipped with an energy harvester and an ESD

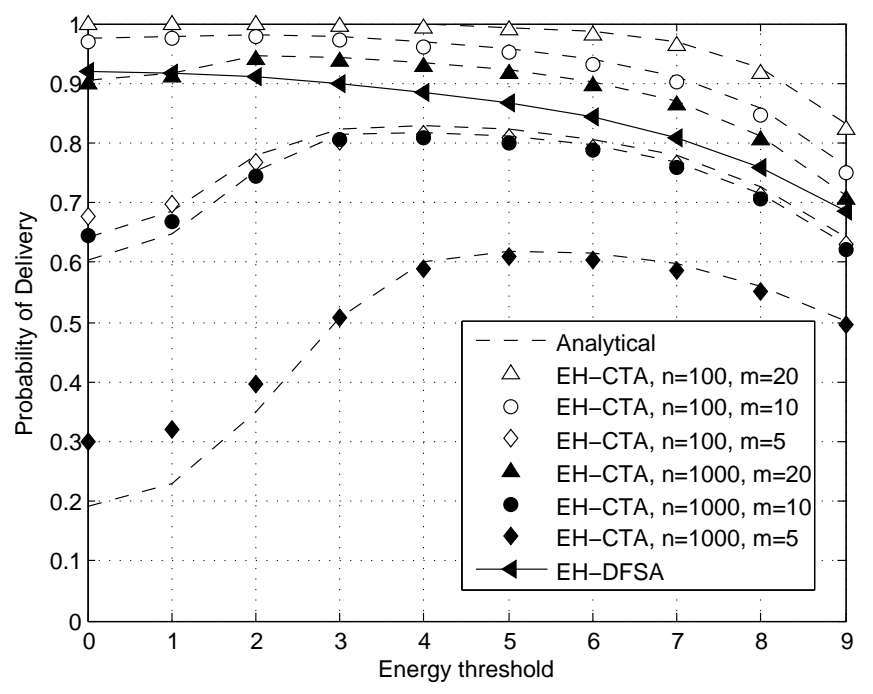

Figure 6: Probability of delivery as a function of the energy threshold.

with a capacity of $N=10$ energy units. We assume that the energy harvested by an end-device during the time period between two consecutive DCRs follows a binomial distribution with a probability mass function given by

$$
q_{j}=\left(\begin{array}{c}
N_{H} \\
j
\end{array}\right)\left(\frac{\bar{E}_{H}}{N_{H}}\right)^{j}\left(1-\frac{\bar{E}_{H}}{N_{H}}\right)^{N_{H}-j}
$$

for $j \in\left\{0,1,2, \ldots, N_{H}\right\}$, where $N_{H}=10$ is the maximum number of energy units that can be captured by an energy harvester, and $\bar{E}_{H} \in\left[0 \ldots N_{H}\right]$ denotes the energy harvesting rate.

Results for EH-CTA have been obtained analytically and through computer-based simulations using MATLAB. As illustrated in the following figures, the tight match between analytical and simulation results validate the accuracy of the our proposed analytical model in Section V. Benchmarking results for EH-DFSA have been obtained through computerbased simulations. In particular we consider an ideal EHDFSA protocol in which the number of contenders in each frame is perfectly estimated, and the number of slots per frame is dynamically configured to be equal to the number of contenders per frame in order to minimize the time to resolve the contention in a DCR. The results of 1000 simulation samples have been averaged for each test case.

\section{B. Energy Threshold}

The probability of delivery for EH-CTA and EH-DFSA is represented in Figure 6 as a function of the energy threshold $\varepsilon_{t h r}$. It has been evaluated by considering: $\bar{E}_{H}=3, n \in$ $\{100,1000\}, m \in\{5,10,20\}$ in EH-CTA, and $N=10$. Recall that an end-device becomes active at the beginning of a DCR if the energy available in its ESD is above $\varepsilon_{t h r}$, which guarantees a minimum number $\varepsilon_{t h r}+1$ of packet retransmissions.

As it could be expected, results show that there exists an optimum $\varepsilon_{t h r}$ that maximizes the probability of delivery for EH-CTA. This optimum value is close to the average number 
of frames $\mathbb{E}[d]$ (14) in which an end-device has to contend in a DCR until it succeeds, i.e., $\varepsilon_{t h r} \approx \mathbb{E}[d]$. The value of $\mathbb{E}[d]$ is represented in Figure 5 as a function of the number of enddevices considering $m \in\{5,10,20\}$. As it can be observed in Figure 6, the optimum $\varepsilon_{t h r}$ is close to 5, 4, or 3 energy units when $m$ is 5,10 , or 20 slots, respectively.

Figure 6 shows a slight deviation between analytical and simulation results when the energy threshold is lower than the optimum value (e.g., for $n=1000, m=5$ and $\varepsilon_{t h r}<3$ ). This is due to the fact that our Markov chain is an approximation of the actual model which assumes a negligible number of end-devices that deplete their energy deposits in a DCR.

While the probability of delivery for EH-CTA increases with the energy threshold until it reaches its maximum value, it can be observed that the probability of delivery for EHDFSA does not increase with the energy threshold and it is insensitive to the number of end-devices. This is due to the fact that in EH-DFSA, since we consider that the number of slots per frame is configured to be equal to the number of contending end-devices in every frame, and assuming that there are neither transmission errors nor capture effect, the probability of successful packet transmission for an end-device in a given frame of EH-DFSA is approximately constant $\approx 0.36$ for all the frames [16], [18]. However, as it can be observed in Figure 4, the probability that an end-device succeeds in a given frame of level $d$ in EH-CTA is very low in the first levels of the contention tree, due to the high probability of collision, and it is much higher than 0.36 when the level number $d$ increases. For example, when $m=10$ slots and $n=10 \cdot m$ end-devices, the probability that and end-device succeeds in one frame of level 2 and 3 is 0.4 and 0.9 , respectively.

Finally, as it can be observed in Figure 6, the probability of delivery for EH-CTA and EH-DFSA decays dramatically when the energy threshold increases above a certain value. Indeed, when the energy threshold is too high, the activation probability decreases, thus reducing the probability of delivery.

\section{Number of Slots}

The probability of delivery and the time efficiency, for EHCTA and EH-DFSA, are depicted in Figures 7 and 8, respectively, as a function of the number $m$ of slots per frame (from 2 to 40 slots). The performance metrics have been evaluated by considering: $n \in\{100,1000\}, \bar{E}_{H} \in\{0.25,0.5,2,4,8\}$, and $N=10$. For EH-CTA, the value of the energy threshold has been set to $\varepsilon_{t h r}=6$ for $m<5, \varepsilon_{t h r}=5$ for $m=5$, $\varepsilon_{t h r}=4$ for $5<m \leq 10$, and $\varepsilon_{t h r}=3$ for $10<m \leq 40$. On the other hand, for EH-DFSA it holds $\varepsilon_{t h r}=0$ and the number of slots per frame is dynamically configured to be equal to the number of contenders in every frame.

Figure 7 shows that the probability of delivery for EHCTA increases when the number of slots per frame increases. This can be explained from the fact that the probability $p_{d}$ of successful transmission in a frame of level $d$ of the contention tree increases with increasing $m$, which in turn results in a decrease in the average number of retransmissions and the consumed energy per end-device. Thus, the probability of delivery tends to improve.

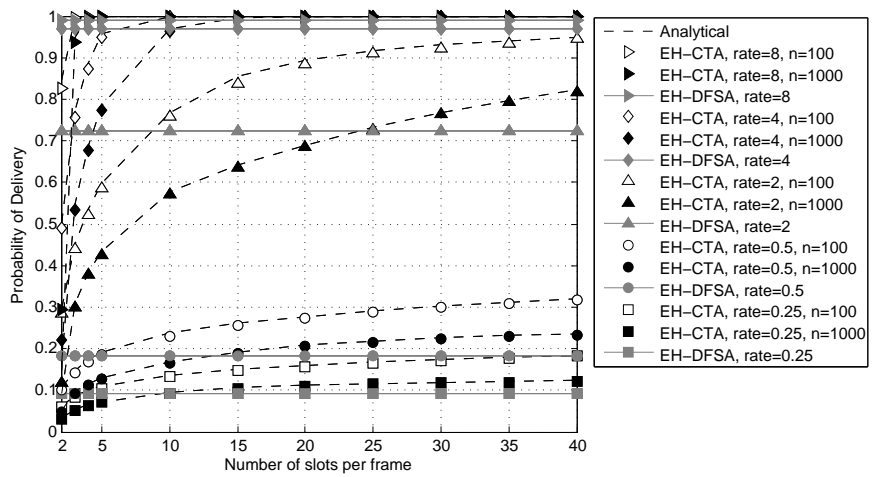

Figure 7: Probability of delivery as a function of the number of slots per frame.

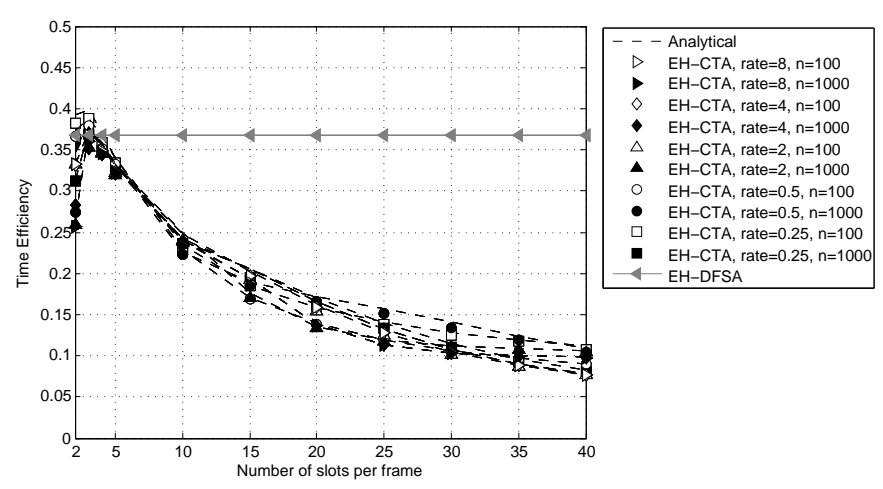

Figure 8: Time efficiency as a function of the number of slots per frame.

We can also observe that the probability of delivery for EHCTA and EH-DFSA increases with the energy harvesting rate. Indeed, the higher the number of available energy units in the ESDs, the higher the number of end-devices that turn to active mode in a DCR which in turn leads to a higher number of possible packet retransmissions. In addition, the probability of delivery for EH-CTA decreases as the number of end-devices increases due to the resulting higher probability of collision. However, it can be seen that the probability of delivery for EH-DFSA is insensitive to the number of end-devices since the number of slots is configured to be equal to the number of contending end-devices in every frame.

As it can be observed in Figure 7, EH-CTA achieves a superior performance compared to EH-DFSA in terms of probability of delivery, for any energy harvesting rate and number of end-devices, if the number of slots per frame in EH-CTA is properly adjusted. For example, if $n=100$ and $\bar{E}_{H} \in\{0.25,0.5,2,4,8\}$, then the value of $m$ in EHCTA must be equal or greater than $4,5,8,7$ and 3 slots, respectively; and if $n=1000$ and $\bar{E}_{H} \in\{0.25,0.5,2,4,8\}$, then the value of $m$ in EH-CTA must be equal or greater than $10,15,25,10$ and 4 slots, respectively.

Figure 8 illustrates that the time efficiency for EH-CTA is maximized for $m=3$ slots, $\eta_{t} \approx 0.38$, whereas it tends to degrade as the number of slots per frame increases. Indeed, while the number of frames required to resolve the contention in EH-CTA tends to its minimum value as the number of slots 


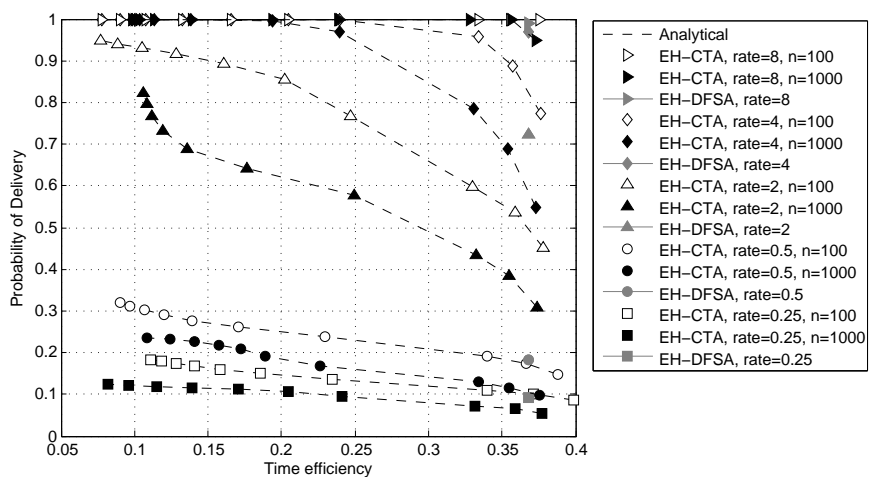

Figure 9: Trade-off between the probability of delivery and time efficiency.

increases, the delay is minimized for $m=3$ slots regardless of the number of end-devices that contend in the DCR [7]. In addition, EH-CTA is slightly better than EH-DFSA in terms of time efficiency at $m=3$ and achieves a very similar performance for different energy harvesting rates and numbers of end-devices.

The trade-off between probability of delivery and time efficiency is investigated in Figure 9 considering a different number of slots per frame $m \in\{3, \ldots, 40\}$ in EH-CTA. It can be seen that as the number of slots per frame increases in EH-CTA, a higher number of end-devices can eventually succeed in their data packet transmissions in a DCR; hence, the probability of delivery increases at the cost of reducing the time efficiency and the data collection rate. In addition, as seen in Figures 7 and 9, for low (e.g., 0.25) and high (e.g., 8) energy harvesting rates, the number of slots per frame in EHCTA can be adjusted to a very low value without a negative impact in the probability of delivery, and increase the time efficiency to a value close to the maximum. However, for intermediate values of the energy harvesting rate (e.g., from 0.5 to 4 energy units), the number of slots per frame in EHCTA needs to be set to a value that depends on the total number of end-devices and the harvesting rate. This can be easily implemented in EH-CTA by including in the RFD and FBP packets a specific field to inform about the frame length to be used in every DCR.

In addition, while the number of slots per frame in EHDFSA must be adapted to the number of contenders in every frame, thus requiring very long frames in highly dense networks, results show that EH-CTA can outperform EHDFSA even using a short and fixed frame length in EH-CTA, i.e., $n / m \gg 1$. Therefore, EH-CTA facilitates scalability and synchronization as the total number of end-devices increases.

\section{Energy Harvesting Rate}

The probability of delivery for the EH-CTA and EH-DFSA protocols is illustrated in Figure 10 as a function of the energy harvesting rate $\bar{E}_{H}$. It has been evaluated by considering: $n \in$ $\{100,1000\}, m \in\{10,20\}$ in EH-CTA, $N=10$, and the optimum energy thresholds.

Results show that the probability of delivery for EH-CTA and EH-DFSA increases almost linearly with the energy har-

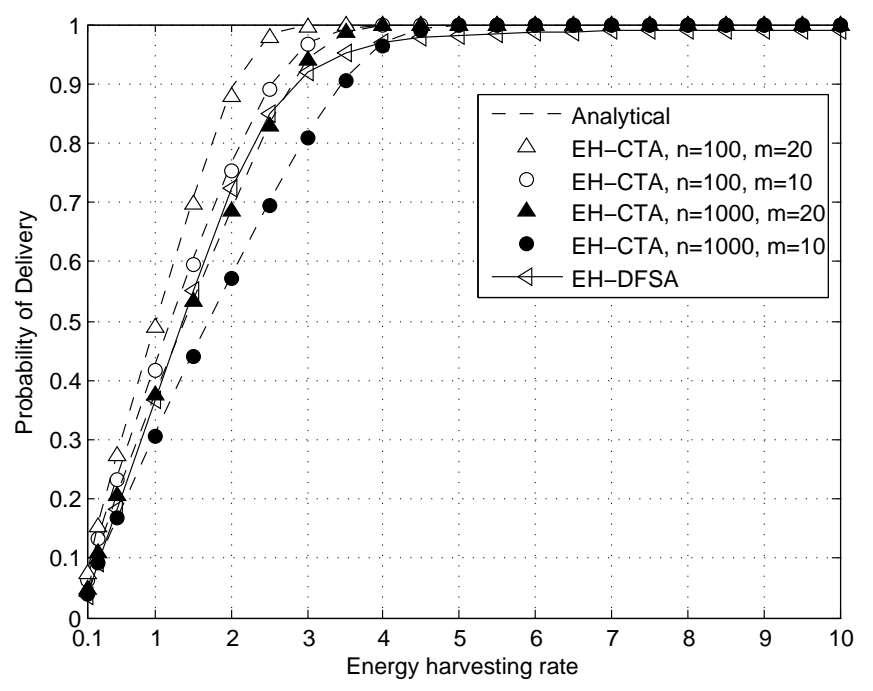

Figure 10: Probability of delivery as a function of the energy harvesting rate.

vesting rate. Naturally, the higher the number of available energy units in an ESD of an end-device, the higher the number of possible retransmissions. In addition, it can be observed that the slope of the probability of delivery for EH-CTA is higher when the number of end-devices is low and the numbers of slots increases. Indeed, as the probability of collision is lower, the energy consumption due to retransmissions is reduced, thus increasing the probability of delivery.

As it could be expected, EH-CTA provides a probability of delivery equal to 1 when the energy harvesting rate is above the optimum energy threshold, i.e., 4 or 3 energy units when $m$ is 10 or 20 slots, respectively, and it outperforms the probability of delivery provided by EH-DFSA for $n \in\{100,1000\}$ when the number of slots in EH-CTA is $m=20$. Indeed, since the tree splitting algorithm organizes the end-devices into sub-groups to reduce the probability of collision in every level of the tree, the average number of retransmissions is lower in EH-CTA than in EH-DFSA, and EH-CTA allows that the data packets of the active end-devices can be eventually transmitted with a finite number of retransmissions and energy consumption.

Results show that EH-CTA with $m=20$ requires lower energy harvesting rate than EH-DFSA to get the same probability of delivery. For example, while EH-DFSA requires $\bar{E}_{H} \approx 5$ to obtain $p_{\text {delivery }}^{S S} \approx 0.98$, EH-CTA requires $\bar{E}_{H} \approx 2.5$ and $\bar{E}_{H} \approx 3.5$ if $n=100$ and $n=1000$, respectively, which means a reduction of $50 \%$ and $30 \%$ in energy harvesting rate. Consequently, EH-CTA allows reducing the total time between consecutive DCRs and thus increases the network throughput.

\section{E. Number of End-Devices}

The probability of delivery for EH-CTA and EH-DFSA is represented in Figure 11 as a function of the total number $n$ of end-devices (ranging from 10 to 1000) in the network. It has been evaluated by considering: $\bar{E}_{H} \in\{2,3,4,5\}, m=20$ in EH-CTA, and $N=10$. 


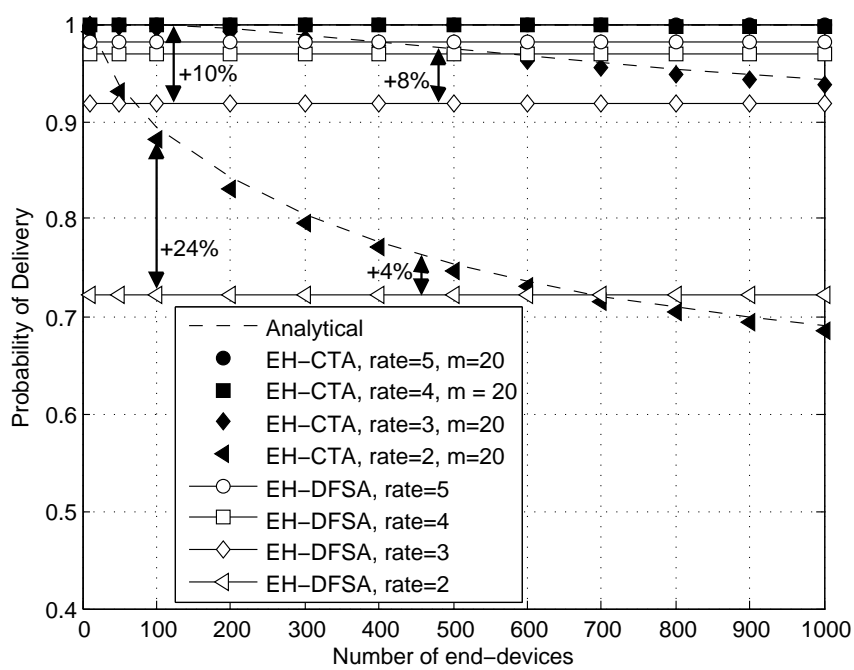

Figure 11: Probability of delivery as a function of the number of end-devices.

As it could be expected, results show that the probability of delivery for EH-CTA decays exponentially with the number $n$ of end-devices. Indeed, the higher the number of enddevices, the higher the probability of collision due to heavier contention, and the higher the probability of energy shortage. It can be observed that if the energy harvesting rate is $\bar{E}_{H}=2$, the probability of delivery for EH-CTA decays rapidly when the value of $n$ increases up to 700. However, if $\bar{E}_{H} \geq 3$, the probability of delivery for EH-CTA decreases much more slowly with $n$. Indeed, a higher energy harvesting rate compensates the higher energy consumption due to a large value of $n$. Results show that when the number of slots per frame in EH-CTA is $m=20$, EH-CTA outperforms the probability of delivery provided by EH-DFSA for $n \leq 1000$. For example, if $\bar{E}_{H}=2$, EH-CTA provides a gain of $24 \%$ in probability of delivery when $n=100$, and a gain of $4 \%$ when $n=500$. If $\bar{E}_{H}=3$, EH-CTA provides a gain of $10 \%, 8 \%$ and 3\% when $n=100, n=500$, and $n=1000$, respectively. If $\bar{E}_{H}=4$ or $\bar{E}_{H}=5$, EH-CTA provides a gain of $4 \%$ or $2 \%$, respectively, for $10 \leq n \leq 1000$.

\section{CONCLUSIONS}

In this paper, we have focused on data-collection scenarios where a large number of end-devices equipped with energy harvesters periodically transmit data to a gateway. While treesplitting algorithms have been identified in the past as a good alternative to improve the performance of random access, their evaluation on networks with energy harvesting (EH) has never received attention. Motivated by this, we investigate the performance of an EH-aware Contention Tree-based Access (EH-CTA) protocol. In particular, we analyze the evolution of the available energy in an end-device and evaluate the probability of delivery, which measures the ability of the protocol to successfully deliver data from the end-devices without entering in energy shortage, and the time efficiency (or data collection rate).
Results reveal the trade-off between both performance metrics and how they are influenced by the number of slots per frame, the energy harvesting rate and the number of end-devices. The time efficiency for EH-CTA is maximized for $m=3$ slots, but degrades as the number of slots increases. Nevertheless, for a high number of slots per frame, an increasing number of end-devices can eventually achieve successful data packet transmission to the gateway, which leads to improved probability of delivery. In addition, results show that with very low and very high energy-harvesting rates, EH-CTA can be adapted to a very low number of slots, without significant effect on the probability of delivery, and increase the time efficiency to a value close to the maximum. However, for intermediate energy-harvesting rates, the number of slots per frame needs to be configured to a value that depends on the number of end-devices and the harvesting rate.

We have compared the performance provided by EH-CTA with that of a benchmark EH-aware Dynamic Frame SlottedALOHA (EH-DFSA) protocol. Results show that EH-CTA achieves a superior performance over EH-DFSA in terms of probability of delivery, for any energy-harvesting rate and number of end-devices, if the number of slots in EH-CTA is properly configured. For example, with 1000 in total enddevices and energy-harvesting rates of $0.25,0.5,2,4$ or 8 energy units, the number of slots per frame in EH-CTA must be equal or greater than $10,15,25,10$ or 4 slots, respectively, to improve the probability of delivery with respect to EH-DFSA. Results show that EH-CTA with 20 slots per frame requires lower energy-harvesting rate to get the same probability of delivery provided by EH-DFSA. Therefore, EHCTA allows reducing the time between data-collection rounds and increases the throughput with respect to EH-DFSA.

Furthermore, while in EH-DFSA an estimation of the number of contending end-devices is required in every frame to properly adjust the used frame length, EH-CTA uses a fixed frame length. While the frame length of EH-CTA can be very short, the frame length of EH-DFSA must be as high as the number of contending end-devices, thus resulting in scalability issues as the number of end-devices increases. Taking that into account, we believe that EH-CTA constitutes a promising alternative for large-scale data-collection scenarios with nodes equipped with energy harvesting capabilities. Future work aims to enhance the presented analysis by including transmission errors due to the wireless channel and the capture effect.

\section{ACKNOWLEDGMENT}

This work was supported by the Research Projects ADVANTAGE (FP7-MCA-607774), GEOCOM (TEC201127723-C02-01), CellFive (TEC2014-60130-P), P2P-SmarTest (H2020-646469), and by the Generalitat de Catalunya under Grant 2014 SGR 1551.

\section{REFERENCES}

[1] S. Sudevalayam and P. Kulkarni, "Energy harvesting sensor nodes: Survey and implications," IEEE Communications Surveys Tutorials, vol. 13, no. 3, pp. 443-461, September 2011. 
[2] L. Kleinrock and F. A. Tobagi, "Packet switching in radio channels: Part 1: CSMA modes and their throughput-delay characteristics," IEEE Trans. Commun., vol. 23, pp. 1400-1416, 1975.

[3] J. I. Capetanakis, "Tree Algorithms for Packet Broadcast Channels," IEEE Transactions on Information Theory, vol. 25, no. 5, pp. 505-515, Sept. 1979.

[4] H. Wu, Y. Zeng, J. Feng, and Y. Gu, "Binary tree slotted ALOHA for passive RFID tag anticollision," IEEE Transactions on Parallel and Distributed Systems, vol. 24, no. 1, pp. 19-31, January 2013.

[5] Y. Xinqing and L. Xuemei, "Evaluating the energy consumption of RFID tag collision resolution protocols," in International Conference on Mobile Ad-hoc and Sensor Networks, February 2010, pp. 215-221.

[6] V. Namboodiri and L. Gao, "Energy-aware tag anticollision protocols for RFID systems," IEEE Transactions on Mobile Computing, vol. 9, no. 1, pp. 44-59, January 2010.

[7] F. Vazquez-Gallego, L. Alonso, and J. Alonso-Zarate, "Energy and Delay Analysis of Contention Resolution Mechanisms for Machine-to-Machine Networks based on Low-Power WiFi," in IEEE International Conference on Communications (ICC), June 2013.

[8] M. Moradian and F. Ashtiani, "Throughput analysis of a slotted alohabased network with energy harvesting nodes," in IEEE International Symposium on Personal Indoor and Mobile Radio Communications (PIMRC), September 2012, pp. 351-356.

[9] V. Sharma, U. Mukherji, and V. Joseph, "Efficient energy management policies for networks with energy harvesting sensor nodes," in Annual Allerton Conference on Communication, Control, and Computing, September 2008, pp. 375-383.

[10] G. Yang, G.-Y. Lin, and H.-Y. Wei, "Markov chain performance model for ieee 802.11 devices with energy harvesting source," in IEEE Global Communications Conference (GLOBECOM), December 2012, pp. 5212 5217.

[11] O. Briante, A. M. Mandalari, A. Molinaro, G. Ruggeri, F. VazquezGallego, and J. Alonso-Zarate, "Duty-Cycle Optimization for Machineto-Machine Area Networks based on Frame Slotted-ALOHA with Energy Harvesting Capabilities," in European Wireless, May 2014.

[12] F. Iannello, O. Simeone, and U. Spagnolini, "Dynamic Framed-ALOHA for Energy-Constrained Wireless Sensor Networks with Energy Harvesting," in IEEE Global Telecommunications Conference (GLOBECOM), December 2010, pp. 1-6.

[13] F. Iannello, O. Simeone, P. Popovski, and U. Spagnolini, "Energy groupbased dynamic framed ALOHA for wireless networks with energy harvesting," in Annual Conference on Information Sciences and Systems (CISS), March 2012, pp. 1-6.

[14] F. Iannello, O. Simeone, and U. Spagnolini, "Medium Access Control Protocols for Wireless Sensor Networks with Energy Harvesting," IEEE Transactions on Communications, vol. 60, no. 5, pp. 1381-1389, May 2012.

[15] F. Vazquez-Gallego, L. Alonso, and J. Alonso-Zarate, "Energy harvesting-aware contention tree-based access for wireless Machine-toMachine networks," in IEEE International Conference on Communications (ICC), May 2016, pp. 1-6.

[16] F. Vazquez-Gallego, J. Alonso-Zarate, S. Wu, Y. Chen, and K. Chai, "Analysis and performance evaluation of dynamic frame slotted-ALOHA in wireless Machine-to-Machine networks with energy harvesting," in IEEE GLOBECOM Workshop on Green Broadband Access, December 2014.

[17] F. Schoute, "Dynamic frame length aloha," IEEE Transactions on Communications, vol. 31, no. 4, pp. 565-568, Apr 1983.

[18] V. Namboodiri, M. DeSilva, K. Deegala, and S. Ramamoorthy, "An extensive study of slotted Aloha-based RFID anti-collision protocols," Elsevier Computer Communications, vol. 35, no. 16, pp. 1955-1966, 2012. 